\title{
Classifications and Development Frameworks of Tourist Resorts Based on Geospatial Division Theory: A Case Study of Sichuan Province, Western China
}

\author{
Jian-Xiong QIN ${ }^{1, a}$, Min-Min ZHANG ${ }^{2, b}$, Jia-Cuo CHENLAI ${ }^{2, c}$ \\ ${ }^{1}$ College of tourism \& historical culture, Southwest University for Nationalities, Chengdu 610041, \\ Sichuan, China \\ ${ }^{2}$ doctor of Southwest University for Nationalities, Chengdu 610041, Sichuan, China

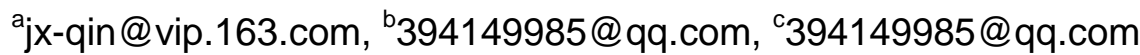

\begin{abstract}
Keywords: Tourist Resorts, Genetic Classifications, Spatial Distribution, Development Model,
\end{abstract} Sichuan Province.

\begin{abstract}
This paper suggests that natural condition and ecologic environment and spatial dimensions are the first important factor. Different kind of tourism resort locates at various districts with different characteristics of natural setting and ecologic environment within Sichuan Province, western China. Different tourism resort of different kinds of genetic possesses different regularities of spatial distribution Based on the comprehensive analysis of geological setting and natural environment in conjunction with ecologic condition etc within Sichuan province not only the genetic classification and environment, but the spatial distribution for different kinds of genetic tourist resorts as well have been discussed. Tourist resorts in Sichuan have been divided into following nine genetic types, including. mountainous-, forest-, hot spring-, lakeside/riverside-, sunshine-, ancient town-, urban-, village-, and integrated-types. Finally, various functional framework characteristics and development construction model of tourist resorts in Sichuan Province have also been built in this paper.
\end{abstract}

\section{Analysis on the Natural Environment of Sichuan Province for Vacation Tour}

Sichuan Province is on the transition zone between the mountains in the western of China and the eastern plains, except the environmental conditions for coastal resort, it has the natural environment and resources required by a variety of vacation tours on the earth. Affected by specific latitude, elevation, topography, circulation, monsoon, temperature, sunlight, rainfall and other comprehensive factors, it presentsexclusive, colorful, unique resource types and characteristics for vacation tour in Sichuan province.

In terms of topography, Sichuan can be divided into the western alpine and plateau region, the eastern basin and mountainous region. Thereinto, the western alpine and plateau region includes the northwesthummocky plateau region and the south-central Hengduan Mountains region (high mountains and deep valleysinterdigitate), which is suitable for special plateau mountain vacation tour; the eastern basin and mountainous region in Sichuan includes the Sichuan basin plain and hilly region $(200-750 \mathrm{~m})$ and the mountainous region in the southwest Sichuan(the lower and middle mountains, $1000-1500 \mathrm{~m}$ ), and the basin is surrounded by mountains, the altitude of mountains is generally $1500-2000 \mathrm{~m}$, which makes for a variety of vacation tours.

In terms of climate conditions, with the Qinghai-Tibet Plateau and mountains around the basin as the barrier for cold air from the north, the winter climatic conditions in Sichuan are more favorable for vacation tour relative to the middle and lower reaches of Yangtze River of the same latitude. Affected by topography conditions, the climate type distribution is diverse in Sichuan Province, for example, subtropical climate zones in the centre of the basin, the vertical mountain climate in the mountains around the basin, and the natural landscape is the subtropical climate type obviously, which makes for vacation tours in different seasons. It features especially the winter sunshine climate resources represented by Panzhihua, Liangshan. Panzhihua belongs to the three-dimensional climate type of the south subtropical latitudinal zone where there are long sunshine duration, strong 
solar radiation, no winter throughout the year and long frost-free period, is known as the natural "big greenhouse": the summer temperatures are not high here, and the average temperature in the hottest month is about $26^{\circ} \mathrm{C}$. In general, the climate features in Panzhihua are: dry and hot spring, damp and heat summer, cool autumn and warm winter, seasons without distinct characteristics, it is perfect for the winter sun vacation tourism, to construct a unique vacation tour type in Sichuan.

Due to a variety of factors, the interaction of temperature, sunlight, humidity, etc. resultsin a variety of weather conditions and climate resources in Sichuan where there are well-developed vegetation, beautiful environment and good ecological environment. Neither the cold winter in the North nor the hot summer in the South is there, and the climate in Sichuan is a prominent three-dimensional climate, fairly mild in winter and in summer, pleasant, and there is a long comfort period. Except the ocean beaches and islands, all important tourist resort attractions could be found in SichuanProvince: the beauty of the mountains and rivers, forest oxygen bar, beautiful rivers, the lake waterscape, hot spring health center, alpine skiing, winter sun, rural countryside and the ancient town recreation.

The unique geographic background and natural conditions have resulted in a three-dimensional environment and diverse cultural ecology in Sichuan, and formed a variety of unique tourism resources in Sichuan which include eight world heritage, two world geological parks, nine national nature reserve, eight national geological parks, eight national scenic area, two national 5A-class scenic spots, thirty four national 4A-class scenic spots, eighteen national forest parks, seven Chinese historical and cultural city, seventeen China excellent Tourism City and one hundred and two national key cultural relics protection units. In different seasons and regions, these climatic factors result in diverse vacation tour zones of different intensities, which are ideal destinations for leisure travelers.

\section{Genetic Classification and Characteristics of Tourist Resorts Sichuan}

In addition to the traditional ancillary facilities, a tourist resort should be strict with especially the condition of the natural environment, which refers to the geological environment, topography, natural safety, soil, air, vegetation, environment and water quality and other conditions. Ecological environmental conditions are the indispensable foundation for a tourist resort, which not only determine the tourist comfort in the resort, and can be directly transformed into holiday products. Considering the nature and safety conditions, attractions, the topography, environmental conditions, land utilization conditions, weather conditions, cultural environment and other general conditions, tourist resorts in Sichuan can be divided into the following nine genetic types. Different types of tourist resorts have varied environmental characteristics.

\section{Mountainous Resort}

This type refers to the tourist resort located in mountainous area which mainly depends on the natural resources in mountains, namely, takes natural tourism resources in mountains as the attractions, the tourism infrastructure in mountainous region as the medium to provide a comprehensive leisure-oriented tourism destination for tourists. The major tourism environment carrier of mountain tourism is the mountainous natural environment, and the core tourism resources include complex mountain landscapes, various mountain water bodies, many flora and fauna landscapes, mountainous three-dimensional climate landscape, so in mountainous resort there usually have been beautiful natural landscapes, good ecological environment and pleasant climatic conditions. The resort projects mainly include the mountain climbing, adventure trips, investigation, field development and other tourism special tourist projects, which is a modern form integrating mountain sightseeing, leisure, fitness, entertainment, education, sports.

\section{Hot Spring Type Resort}

Taking the hot springs as the core, this type resort is a regional complex which integrates the surrounding natural environment and community, gives full play to the material composition in the hot spring water and their natural functions such as hydrotherapy and health care, where tourists can 
recuperate. A hot spring resort should be set at the place where a hot spring flows out and the temperature, flow, salinity, etc. reach a certain standard, if the natural environment and socio-economic conditions allow, aiming at recuperation, recovery, physical therapy, rehabilitation, health care and the future market.

Hot spring resorts are generally located in geological regions where there are relatively active tectonic activities, mainly located in mountainous and hilly areas. It features the mountainous topography with a variety of climatic conditions. Particularly the hot springs - winter - ecological environment are the best combination, hot spring factors include the temperature, flow, salinity, etc. Most hot spring resorts are located in the lovely natural environment, where there are not only high-end health center, modern rehabilitation equipment, clinics, nursing homes and treatment facilities. It can provide professional infirmary doctors, comfortable accommodations, first-class food, etc. and modern recreational tourism projects.

\section{Forest Type Resort}

Forest landscape resources are the core of this type tourist resort which makes use of the excellent forest resources and forest environment to develop and construct the tourist facilities, provide summer vacation, health vacation and other relevant tourism services. This type resort usually integrates with mountains, lakes or rivers to improve the quality. As for forest leisure vacation, the accommodation in forest is the main mode, travel through the forest is the main recreation way, to enjoy the forest environment is the main purpose; the forest is one of the best places away from the bustle of the city, whose market potential should not be underestimated. Forest leisure vacation is a dispersive type of leisure. The overall distribution is wide, and the range of leisure activities is also dotted. It mainly depends on forest parks, nature reserves, scenic areas, takes advantage of its rich landscape resources (nature, culture) to provide high-quality tours, vacation, leisure, health care nursing, science education, culture and entertainment of a certain scale, and other activities. For forest leisure vacation, forest convalescence vacation, forest leisure and sports vacation, etc., there are some essential vacation items such as forest shower, roam in the forest, forest hiking, probing, adventure, forest fishing, forest hunting, forest village tour, field collection, cookout, picnics, etc. Diverse forest recreation projects include forest golf, forest horse racing, forest skiing and other less-known projects.

\section{Ancient Town Type Resort}

The unique geological features and geographical conditions of Sichuan gave birth to long-standing Bashu cultural tourism towns. Sichuan has more than 4,000 ancient towns, 14 of which have been selected to be the national famous historical and cultural towns, accounting for 9.8\%. Some tourism development has been conducted in Luodai, Li Zhuang, Huanglongxi, Pingle ancient towns, etc., which have attracted lots of domestic and foreign tourists, and achieved good economic and social benefits. Relying on the rich cultural heritage, with the harmony of mountain water - town and the perfect blend of nature and human, combined with the excellent environment and livable condition, ancient towns should be equipped with tourism service facilities to be the integrated tourism where visitors and tourists can have cultural education, medical services, administrative services, entertainment, leisure and other services. Ancient town type resort is unique resort type in Sichuan or even China with core competitiveness. In Sichuan Province, there are always optimal topography for variety of geological and geomorphological background, the ancient town vacation tour modes include: ancient town leisure, health living, weekend vacation, which take the ancient town landscape, the ancient town culture, the ancient town natural environment as the core attraction and carrier.

\section{Village Type Resort}

Unique geological conditions in Sichuan gave birth to unique idyllic countryside landscape in Sichuan, Dujiangyan Irrigation Project for the Minjiang River gave birth to the land of abundance with a long history, and the unique natural environment and local culture created a distinctive countryside vacation conditions in Sichuan. The village type resort and the urban resort complement 
each other. Village-type resort is an original eco-tourism and leisure world which is consisted of rustic environment, rural landscape, rural customs, life and production in village. It is an operating form of vacation tour with sightseeing, recreation and fitness, leisure and other functions, which has clear geographical scope and the unified management and operation, a relatively independent resort community that can provide leisure facilities and services. It has distinct characteristics of village: the authenticity, synthesis and ecology lie in the agricultural production, agricultural activities, farm buildings, customs, the lifestyle and other material and non-material forms of village, for example, the village resort in the front hill of the Longmen Mountain and the rural leisure vacation area around Chengdu. Currently, the Chengdu International Village Resort takes the "cultural tour, romantic garden" as the theme, ecology, culture, leisure, vacation, health as the main topics, Gu Shu Yuan, Lao Lin Pan, Man Shenghuo, Jin Shi Jiang as the basis to promote the regional leisure vacation and the amusement theme aggressively, and further expand the popularity, reputation and influence, which creates a famous and international village-theme recreation area and a world-class travel destination.

\section{Lakeside / Riverside Type Resort}

In a "province with thousands of river". Sichuan, rivers go from west to east, lakes run from north to south. In addition to the White River in northwest Sichuan, Black river, the Jinsha River, etc., in Sichuan Province there are some famous rivers such as Yashe River, Minjiang River (including Dadu, Qingyi), the Tuo River and Jialing River (including An Jiang, Liang Jiang, Chishui River and other rivers, where rivers and lakes are dense. It is an ideal province for Lakeside / riverside type vacation. A lake tourism resort refers to a tourism destination which takes the natural lakes or artificial reservoirs as the major landscape. Since China began to set up tourist resort in 1992, more than $1 / 3$ belong to the lake tourist resort which has been the most important tourism resort type in China. It makes use of lake resources, creates a good environment and chooses an advantageous location to meet the recreation and leisure needs of travelers. It is a kind of resort area where visitors can enjoy the recreation in water, a fishing vacation and other leisure services and facilities. In addition to the general characteristics of tourist resort, the lake tourist resort has some features different from other types due to the lake resources. In the lake resort area, there are specific resources, integrated tourism functions, discrete layout features by the lake, the landscape of high sensitivity and ecologically fragile areas.

\section{Urban Resort}

This type refers to especially the provincial capital Chengdu. As a "World Heritage City", Chinese culture preserve, the Chinese leisure city, the birthplace of rural tourism, Southwest Business Exhibition Center, Chengdu is not only rich in city tourism resources, but also has a long comfort period for travel and excellent weather conditions for holiday, which are the important climate basis for the construction of urban tourism resort, on the topography, this type of resort is consisted of mainly the plains and a few hilly areas; on the region of space, it is a modern urban area surrounded by rural countryside, therefore, its climate seems like an indoor artificial climate in the urban concrete jungle. Artificial urban environment, garden space, urban ecological environment, urban artificial landscape and urban human activity resort. Since the urban resorts are located in the metropolitan cities, transportation, communications, utilities and materials are always available, on the basis of the developed service facilities in the metropolis, the urban resort would be a fashion, popular, modern resort, which can provide a urban eco-holiday, residence, exhibition, business, conferences, festivals and other services. The urban type and village-type resorts complement each other.

\section{Sunshine Type Resort}

This type refers in particular to Panzhihua, Xichang and the surrounding areas, PanzhihuaPingba is at an altitude of no more than 1500 meters, a river valley, where the annual average temperature is $19.7-20.9^{\circ} \mathrm{C}$, the annual sunshine duration is $2352-2737 \mathrm{~h}$, the annual solar radiation is $5600-6300 \mathrm{mj} / \mathrm{m}^{2}$, the accumulated temperature $\geq 10^{\circ} \mathrm{C}$ is $1979-5479^{\circ} \mathrm{C}$ more than the average level in 
Sichuan or in the western regions. Relying on its unique climatic conditions, especially relying on the unique characteristics and conditions of "warm winter, sunny winter" (rich sunshine, strong solar radiation, warm climate) combined with mountain and river views, customs, hot spring, "off-season" fruits and flowers, the resort has been equipped with related tourism facilities, to build the sunshine eco-tourism resort in China, provide tourists with aintegrated tourism destination for sunshine and ecological vacation tour, health tourism, sports and leisure, sightseeing and other services. It could be a high-end and high-quality sunshine national tourist resort. It can make use of its "off-season" environment and resources to provide services like the summer vacation from south to north, the winter vacation from north to Sanya, and the difference is it has unique warm-winter mountain climate and the sunshine in winter, and off-season fruits and flowers and other agricultural resources due to the subtropical climate and mountains.

\section{Integrated Resort}

This type is a major type of tourist resort at home and abroad. In Sichuan Province, this type is located mainly in mountainous and hilly regions, which always integrates at least two elements as follows: mountains, hot springs, forests, lakeside / riverside, sun, ancient town, village, etc. which are important environmental conditions, resources and unique elements for tourist resorts in Sichuan Province. On the basis of two or more resources, it integrates other environmental conditions to develop and construct the tourism facilities, which is leisure-oriented integrated tourism area for tourists, such as mountain forest resort, lakeside/ riverside forest resort, hot spring village resort, sunshine lake resort and ancient town hot spring resort.

\section{Analysis on the Spatial Distribution Pattern of Tourist Resort in Sichuan Province}

Natural conditions, ecological environment and space element make up the first element of tourism resort. Because the natural conditions and ecological environment in different regions of Sichuan Province have their differences, and it forms different genetic types of tourist resorts. The spatial distribution in different genetic types of tourist resort has its own regularity.

Mountain type resort is mainly rely on the natural mountain landscape resources to form the tourist resort, it is basically distributed in the mountainous and hilly region of Sichuan Basin periphery, especially in Ya'an area where is in the southwestern of Sichuan and in Dazhou, Guanyuan, Bazhong, Nan Chong and Guang'an areas where are in the northeast of Sichuan, and the second distribution in Liangshan, Ganzi and Aba areas where are in the west of Sichuan.

Tourist resort of hot spring type is in limited distribution in hot spring development areas of Sichuan Province, in terms of geological conditions it is mainly in Liangshan, Ganzi and Aba regions where is in the west of Sichuan, secondly in Ya'an area where is in the southwest of Sichuan, in the Chengdu Plain surroundings and in Yibin area where is in the south of Sichuan. Hot spring resort area constitutes important type of tourist resort in Sichuan province, and it mainly distributed in Sichuan basin periphery, and till now hot spring resort areas which have been successfully built including Hailuogou, Yulin Palace, Mount Emei, Mount Huashuiwan and Mount Zhougong etc. Sichuan is one of the provinces which have abundant hot springs resource in the country, extending Aba and Ganzi, Liangshan three states for the east side line, it can be divided into western and eastern Sichuan Basin hot spring area. The former is specifically distributed in Ganzi, Aba and Liangshan states, with high hot spring; the latter is mainly distributed in Sichuan Basin periphery, including Dayi, Ya'an, Yibin, Dazhou, Guang'an and other places, with low hot spring. The distribution of Sichuan hot spring is mainly controlled by the tectonic fault distributions of the Fresh Water River, mount Longmen, Jinsha River, Ganzi-Litang, mount Huaying and etc., including the following hot spring enrichment zones:Ganzi-Litang, Yidun-Duocheng,Luhuo-Kangding, Shimian-Dechang, Yuexi-Ningnan,Ebian-Jinyang, Dayi-Anxian and etc.

Forest Resort is a tourist resort area with the forest landscape resources as the core formation, Sichuan province is one of the most provinces in Forest Park, nature reserve, scenic area. Forest Resort depends on the development of forest resources has become one of the most important 
holiday tourism products in Sichuan Province,and it mainly distributed in the area around the Sichuan basin. Including Ya'an area of southwest Sichuan, Ganzi,Aba, Liangshan;and Bazhong, Dazhou, Guangyuan, Guang'an, Nanchong in the northeastern Sichuan; Yibin, Luzhou in the Southeast Sichuan; and the surrounding areas of Chengdu Sichuan belongs to the typical province with enrichment forest resources, and it is also the province with most Forest Parks all over the country. Forest tourism here is characteristic, with great development potential and good condition in Forest Park. Such as Dragon pool, Mount Wawu and other 30 Forest Park have already possessed tourist reception conditions.

Due to the geographical location of Sichuan geology and pleasant ecological environment and climate conditions, the ancient towns have been concentrated in the provincial distribution since ancient times, ancient towns are widely distributed in various regions of Sichuan province, and this forms the different geographical characteristics of Bashu ancient town system. Relying on the characteristics of the rich cultural heritage of ancient town, the harmony of the landscape, and the perfect fusion between man and nature, we construct resort area. For example, there are the ancient city of Langzhong in Nanchong which is located in the middle reaches of Jialing River in Northeast Sichuan hilly area, Anren Liu's Manor in Dayi which is located in Chengdu Plain in the Northwest Plateau of Sichuan transitional zone, Taoping Qiang Village in Lixian which is located in the Mount Longmen fault interrupt alpine valley region, Jiarong Tibetan Village in Danba which is located in the high gorge valley area of Hengduan Mountains, Huanglong Valley Town in Shuangliu County which is located in hinterland of Chengdu Plain, Lugu Lake town in Yanyuan which is located in the southeast margin of Qinghai Tibet Plateau on the West Bank and on the downstream of Yalong River, Shangli ancient town in Ya'an which is located in mountainous area of southwest Sichuan, Li Zhuang ancient town which is located in the south margin of Sichuan Basin and on the upper reaches of the Yangtze River, and the Zhaohua ancient town in Guangyuan which is located in edge of Sichuan Basin and on the upstream of the Yangtze River, and the Baofo ancient town in Hejiang County which is located in south mountain area of Sichuan basin and etc..

Sunlight-type Resort Limited located in Panzhihua, Xichang and its surrounding areas. Just like the south to the north in summer vacation, and the north to Sanya in Hainan province in winter vacation. The difference is here to subtropical climate, mountain area, unique mountain climate in winter and winter sunlight conditions,as well as anti-seasonal agricultural products especially fruits and vegetables and flowers resources. Panzhihua city is located in the south central section of the Panxi rift, the terrain from the northwest to southeast tilt, and mountains are approximately from north to south, the relative elevation is generally at 1000-2000 meters. In the city, the highest point is the main peak of Mount Bailing which is about 4196 meters above sea level, and the lowest point is the ground town of Sizhuang and about 937 meters above sea level. Due to the north, west, east of the barrier layers of tall mountains, the climate of Panzhihua city is unique in the province and even the whole country in the same latitude areas. The climate in Panzhihua city is stereo South-Asia tropical baseband one. Here, winter without cold, and summer without heat, the four seasons here just like spring.

Village and urban leisure types of resort area complement each other, and these compose one of the most important tourism resort type in Sichuan province, including the surrounding countryside resort which is located in the metropolitan area, large resort village along both sides of the channel, village resort with the characteristics of the villages and towns, and mountain ecological village resort and etc.. Because of the unique geological and geomorphologic and geographical background in Sichuan Province, here has a distribution of almost all the genetic types of the rural resort, including the rural-type resort in Gan-A-Liang mountains of Western Sichuan Plateau, the rural-type resort of Piedmont hills surrounding Chengdu, the rural-type resort in the former mountain belt along Mount Micang - Mount Daba and Mount Huaying of northeast Sichuan, the rural -type resort in hilly areas of southeast Sichuan, the rural-type resort of eco-mountain areas in Ya'an of southwest Sichuan, and the rural-type resort of sunny areas in Panzhihua. On the small-scale, there are mainly city-based rural resort, traffic-based rural resort, scenic-based rural 
resort, town-based rural resort, industry-based rural resort; the spatial distribution of different types of rural resort is closely related to its causes of large-scale spatial distribution.

Sichuan has a number of rivers and lakes with a long history, in addition to the beautiful environment, good ecological and pleasant climatic conditions, which lays an important foundation to develop the Riverside-type or Lakeside-type of tourism resort in Sichuan region. Sichuan is known as the "thousand water province", except White River and Black River in the region of Hongyuan, Nuoergai and Aba which belong to the Yellow River water system, all the rest belong to the Yangtze River water system. The main rivers in Sichuan are Yalong River, Jinsha River (including Dadu River and Qingyi River), Min River, Jialing River (including Fu River and Qu River), Tuo River, Red Water River and other rivers, each of them has a length of over 500km; all the rivers have a collection by the edge of the mountains and converge at the bottom of the basin, then inject into the Yangtze river. There are more than a total of 1000 natural lakes in Sichuan. The more famous natural lakes among them are the Qionghai which is located in Xichang (has a water area of 31 square kilometers), the Lugu lake (has a water area of 72 square kilometers, about 27 square kilometers in Sichuan Province), the Horse Lake in Leibo County. In addition to natural lakes, there are some well-known reservoirs or artificial lakes, such as the Longquan Lake in Chengdu, Chaoyang Lake, Sancha Lake in Jianyang and so on. In the rivers and lakes system, the more famous lake-type resort including: Lugu Lake, Sancha Lake, Qionghai in Xichang, Black Dragon Pool, Shengzhong Lake, as well as the related tourism resort along Qingyi River and Jialing River which has been developed into a Lakeside-type or Riverside-type resort.

Urban leisure resorts are those which particularly located in the range of cities or metropolitan areas; relying on city or metropolitan resources and after artificial creation forms the urban ecological leisure resort. It has a complement with the rural leisure resort in space distribution. Usually the rural tourism resort distributes around the city where possesses metropolis leisure resort. On the basis of the topographic features of regional space, urban leisure holiday resorts are mostly distributed in plain areas, maybe as syntheses in cities, and hotels. Parts of them are distributed in hilly mountain cities, such as Kunming, Guiyang, and other leisure resort area in secondary and tertiary cities. From space, the distribution may at the seaside city or in the urban, such as Hangzhou is the city which is surrounded with lakes, Chengdu is the city which is located in the front of mountains, Chongqing is the city which is located in mountains; and Sanya city in Hainan Province which is relying on the difference of climate resources, Harbin ice city in the northland and etc.

The integrated resorts of Sichuan Province are mainly distributed in mountainous and hilly regions around the Sichuan Basin, especially the Ya'an areas in southwest Sichuan, where have the best ecological environment and climate conditions; Mount Micang-Guangwu, Mount Daba, Mount Huaying and the relevant areas in northeast Sichuan, where have the mountain backdrop with Qinba mountain specialties, specialty towns and landscapes; The golden triangle area(Sichuan, Guizhou and Chongqing)in southeast Sichuan, where the ancient town has characteristic of Southern Sichuan features, and where has hilly landscape and landscape resources. Such as mountain forest resort, riverside or lakeside forest resort, hot springs village resort, sun-lake's resort, hot spring and ancient town resort, etc. Mountain background, hot spring resources, forest ecology, lake landscape, climate resources, characteristic villages, pastoral scenery, and all these are comprehensive tourist areas constituted with two and above of resources; and these resources mainly distributed in the mountain background region with good ecological and beautiful environment and pleasant climate, and usually, it is the important condition that the integrated resort relying on.

\section{References}

[1]Alexis Saveriades. Establishing the social tourism carrying capacity for the tourist resorts of the east coast of the Republic of Cyprus. Tourism Management, Volume 21, Issue 2, April 2000, Pages 147-156.

[2]Anya Chapman, Janet Speake. Regeneration in a mass-tourism resort: The changing fortunes of 
Bugibba, Malta. Tourism Management, Volume 32, Issue 3, June 2011, Pages 482-491.

[3]Sasicha Sukkay, Nopadon Sahachaisaeree. A Study of Tourists' Environmental Perceptions of the Functional Design of Popular Resorts in Chiang Rai Province [J]. Procedia-Social and Behavioral Sciences, 2012, 50(5), 114-122.

[4]Hana Ayala. Ecoresort: A Green Masterplan for the International Resort Industry [J]. International Journal of Hospitality Management, 1995, 14(3-4):351-374.

[5]Gerda Priestley, Lluis Mundet. The Post-Stagnation Phase of the Resort Cycle Original Research Article [J]. Annals of Tourism Research.1998, 25(1):85-111. 